\title{
Weight Gain, CTCAE
}

National Cancer Institute

\section{Source}

National Cancer Institute. Weight Gain, CT CAE. NCI Thesaurus. Code C55338.

A finding characterized by an unexpected or abnormal increase in overall body weight; for pediatrics, greater than the baseline growth curve. 